\title{
Characteristic clinical features as an aid to the diagnosis of suppurative keratitis caused by filamentous fungi
}

\author{
P A Thomas, A K Leck, M Myatt
}

Br J Ophthalmol 2005;89:1554-1558. doi: 10.1136/bjo.2005.076315

See end of article for authors' affiliations

Correspondence to:

Correspondence to:
Astrid Leck, PhD,

Department of Infectious

and Tropical Diseases,

London School of Hygiene

and Tropical Medicine,

Keppel Street, London

WCIE 7HT, UK; astrid.

leck@lshtm.ac.uk

Accepted for publication 28 July 2005

\begin{abstract}
Aim: To assess whether the presence of characteristic clinical features can be used as a diagnostic aid for suppurative keratitis caused by filamentous fungi.

Methods: Patients presenting with suppurative keratitis in India underwent detailed clinical examination followed by microbiological investigation of corneal scrapes. A partial diagnostic score based upon the strength of the association, as estimated by the odds ratio, between reported clinical features and laboratory confirmed diagnoses was devised and subsequently tested using a case series from Ghana. Results: Serrated margins, raised slough, dry texture, satellite lesions and coloration other than yellow occurred more frequently in cases of filamentous fungal keratitis than bacterial keratitis $(p<0.05)$. Hypopyon and fibrinous exudate were observed more frequently in bacterial keratitis $(p<0.05)$. When incorporated into a backwards stepwise logisitic regression model only serrated margins, raised slough, and colour were independently associated with fungal keratitis; these features were used in the scoring system. The probability of fungal infection if one clinical feature was present was $63 \%$, increasing to $83 \%$ if all three features were present.

Conclusions: Microbiological investigations should be performed whenever possible; however, where facilities are not available, a rapid presumptive diagnosis of suppurative keratitis may be possible by scoring clinical features.
\end{abstract}

$\mathrm{S}$ uppurative keratitis is an important cause of monocular blindness worldwide, in particular in developing countries in tropical latitudes. Most ophthalmologists in the developing world do not have recourse to specialised ocular microbiological laboratory facilities and may have to depend on their own clinical acumen for diagnosis, with empirical treatment of patients with these infections. Kaufman and Wood described certain clinical manifestations that they believed to be characteristic of mycotic keratitis, including a severe ocular reaction; folds in Descemet's membrane; "hyphate" margin; firm and elevated lesions; hypopyon; endothelial plaque and immune ring. ${ }^{1}$ However, these descriptions were based on a study of only 15 patients with culture proved fungal keratitis. According to Jones, fungal infection is likely in a suppurative corneal lesion with one or more of the following features: a dry, raised ulcer with crenate, spiculate or pseudohyphate border; satellite lesions; recurrent hypopyon or posterior chamber endophthalmitis with progressive shallowing of the anterior chamber; failure to respond to antibacterial treatment. ${ }^{2}$ These guidelines were based on observations in just 29 patients with laboratory diagnosed fungal keratitis.

Since then these clinical features have generally been accepted as being diagnostic of filamentous fungal keratitis. Keratitis caused by yeast-like fungi such as Candida spp has been described as resembling bacterial keratitis. ${ }^{3-5}$ Recent studies on large numbers of patients with culture proved mycotic keratitis have tended to document only the frequency of occurrence of these features and there has hitherto been no attempt to compare the occurrence of these features in a large series of patients with bacterial and fungal keratitis with a view to assessing their suitability as diagnostic aids for suppurative keratitis. ${ }^{67}$ In this study we have attempted to determine to what extent the presence of certain clinical features may be used to provide a rapid, presumptive diagnosis of suppurative keratitis caused by filamentous fungi.

\section{METHODS}

A prospective study of suppurative keratitis (defined as loss of corneal epithelium with underlying stromal infiltrate and suppuration associated with signs of inflammation, with or without hypopyon) was conducted at three centres in southern India and in three centres in Ghana between June 1999 and May 2001. The aetiology of the infection in 1090 consecutive cases ( 800 from India and 290 from Ghana) has already been reported. ${ }^{8}$

\section{Clinical findings}

Ophthalmologists examined patients at the different centres using a standardised protocol and proforma. A detailed examination was performed on each patient at the slit lamp; clinical features were documented, drawings made for patient records, and a photograph was taken of the affected eye. The length of the slit beam was used to assess the vertical diameter of the corneal ulceration; this was then turned $90^{\circ}$ to assess the horizontal diameter. Using the slit beam parallelopiped the depth of ulceration and infiltrate were assessed. The presence or absence and form of the following clinical features were documented: elevation of slough (raised, flat), texture of slough (wet, dry), ulcer margins (serrated, well defined), satellite lesions, immune ring, hypopyon, keratic precipitates, or perineural infiltrate, fibrin, flare or cells in the anterior chamber (AC), and deep lesions (posterior corneal abscess, endothelial plaque).

\section{Microbiological investigations}

Laboratory diagnosis was determined using microscopy and culture. ${ }^{9}$ Microbial cultures were considered to be significant if (i) growth of the same organism was demonstrated on two or more solid culture media; (ii) there was semi-confluent growth at the site of inoculation or growth on one solid medium consistent with microscopy (that is, appropriate staining and morphology with Gram stain); or (iii) semiconfluent growth at the site of inoculation on one solid 
Table 1 Univariate analysis of clinical features occurring in fungal and bacterial keratitis

\begin{tabular}{|c|c|c|c|c|c|c|c|c|}
\hline Clinical feature & $\begin{array}{l}\text { Frequency } \\
\text { (\% fungal) }\end{array}$ & $\begin{array}{l}\text { Frequency } \\
\text { (\% bacterial) }\end{array}$ & $\chi^{2}$ & OR (Cl) & p Value & Sens & Spec & PPV \\
\hline Serrated margins & 180/228 (79\%) & $63 / 132$ (48\%) & 37.14 & 4.09 (2.57 to 6.56$)$ & 0.00 & 0.79 & 0.52 & 0.74 \\
\hline Raised slough & $135 / 228$ (59\%) & $52 / 132$ (39\%) & 13.50 & 2.23 (1.44 to 3.55$)$ & 0.00 & 0.59 & 0.61 & 0.72 \\
\hline Dry texture of slough & $101 / 228(44 \%)$ & $37 / 132$ (28\%) & 9.36 & 2.04 (1.29 to 3.26$)$ & 0.00 & 0.44 & 0.72 & 0.73 \\
\hline Satellite lesions & $51 / 222(23 \%)$ & $17 / 132$ (13\%) & 4.91 & 1.95 (1.08 to 3.61$)$ & 0.04 & 0.22 & 0.87 & 0.75 \\
\hline Hypopyon & $105 / 219(48 \%)$ & $83 / 128(65 \%)$ & 9.29 & $0.50(0.32$ to 0.78$)$ & 0.00 & 0.48 & 0.35 & 0.56 \\
\hline Fibrin & $21 / 210(10 \%)$ & $28 / 125(22 \%)$ & 9.65 & $0.38(0.20$ to 0.70$)$ & 0.00 & 0.10 & 0.78 & 0.43 \\
\hline Colour (not yellow) & $213 / 228(93 \%)$ & $106 / 132(80 \%)$ & 14.26 & 3.47 (1.77 to 6.98$)$ & 0.00 & 0.93 & 0.20 & 0.67 \\
\hline
\end{tabular}

medium (if bacteria); or (iv) growth of the same organism on repeated scraping. Culture positivity was the "gold standard" used to establish the diagnosis of the bacterial ulcers. If fungal hyphae were observed in corneal tissue, but failed to grow in culture, the causative organism was reported as fungal.

Verbal patient consent was obtained but written consent was not considered appropriate as the study did not involve any deviation from routine diagnostic or treatment protocol. Patients were excluded from the study if they did not agree to investigation and treatment.

\section{Cases included in this study}

In this study only patients with confirmed bacterial or fungal infection were included in the analysis. The following patients were excluded from the 1090 consecutive cases: those with suspected or confirmed viral keratitis, corneas at risk of perforation (where corneal scrapes were not possible) and small ulcers; defined as an epithelial defect $<2 \mathrm{~mm}$ (greatest diameter) and/or an infiltrate of $<4 \mathrm{~mm}$ (greatest diameter) (128) as these comprised early stage corneal ulcers which may present with non-specific signs, and characteristic clinical features may not be present. Those with a mixed infection (44), Acanthamoeba keratitis (seven), unconfirmed laboratory diagnosis (249), or where clinical features had not been adequately recorded (12) were excluded. The clinical features of 360/800 patients in India with confirmed bacterial or fungal infection were analysed further to devise a diagnostic score. The diagnostic score was subsequently applied to 115/290 cases of bacterial or fungal infection from the Ghanaian patient dataset (after excluding patients according to the criteria applied to the Indian dataset).

\section{Analysis of data}

From the Indian dataset pairwise associations between clinical features and diagnosis were investigated using SPSS (SPSS for Windows, Release 10.0.5, SPSS Inc, 1989-2001) and EpiCalc. ${ }^{10}$ Significant associations were entered into a logistic regression model and non-significant associations removed using backwards elimination. A score was created from a count of signs positively and independently associated with a fungal aetiology. An operating characteristic (OC) curve for the diagnostic score was created by computer based

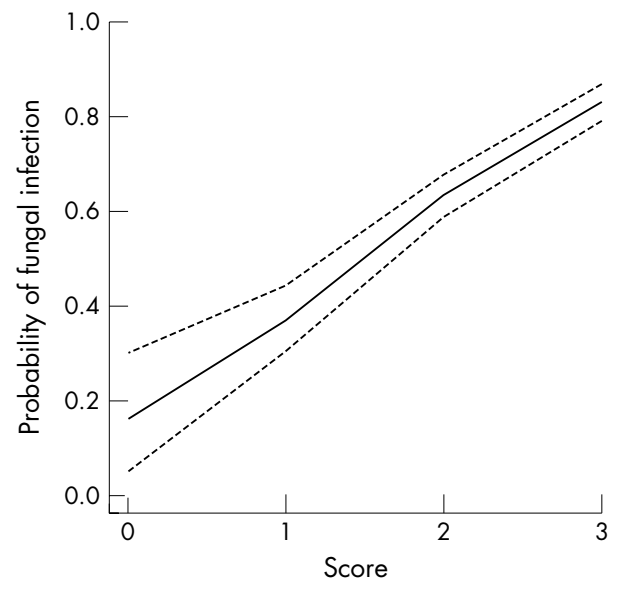

Figure 1 Operating characteristic $(\mathrm{OC})$ curve for diagnostic score (India data).

simulation with the prevalence of fungal infection at each score estimated in 1000 bootstrap (that is, with replacement) samples of size 1000 taken from the clinical dataset. The probability of fungal infection was estimated using the median prevalence found at each score in the bootstrap samples. A 95\% confidence interval around the probability of fungal infection was estimated using the 2.5\% and $97.5 \%$ quantiles of the distribution of the prevalences found at each score in the bootstrap samples. Simulations were performed using the R language for data analysis and graphics. ${ }^{11-13}$ Data were plotted using R. The Ghanaian patient dataset was tested using the diagnostic score devised from the Indian data, and an OC curve was created by computer based simulation, as for the Indian data, and compared.

\section{RESULTS}

Clinical data for 360 patients from India with confirmed fungal (228) and bacterial (132) keratitis were analysed. Features which occurred significantly $(\mathrm{p}<0.05)$ more frequently in fungal than in bacterial corneal ulcers by univariate analysis were as follows: serrated margins, raised slough, dry textured slough, satellite lesions, and colour

\begin{tabular}{lll|}
\hline $\begin{array}{l}\text { Table } 2 \\
\text { keratitis }\end{array}$ & Multivariate analysis of clinical features occurring in fungal and bacterial \\
\hline Clinical feature & OR (CI) & p Value \\
\hline Serrated margins & $3.45(2.12$ to 5.64$)$ & 0.00 \\
Raised slough & $2.32(1.43$ to 3.74$)$ & 0.00 \\
Fibrin & $0.39(0.20$ to 0.77$)$ & 0.01 \\
Colour (not yellow) & $2.85(1.34$ to 6.03$)$ & 0.01 \\
\hline
\end{tabular}


Table 3 Screening test indices at each level OC curve

\begin{tabular}{lllll}
\hline Score & Sensitivity & Specificity & PPV & NPV \\
\hline$>0$ & $0.99(0.97,1.00)$ & $0.08(0.04,0.14)$ & $0.65(0.60,0.70)$ & $0.83(0.51,0.97)$ \\
$>1$ & $0.88(0.83,0.92)$ & $0.41(0.33,0.50)$ & $0.72(0.66,0.77)$ & $0.66(0.54,0.76)$ \\
$>2$ & $0.45(0.38,0.51)$ & $0.84(0.76,0.90)$ & $0.83(0.75,0.89)$ & $0.47(0.40,0.53)$ \\
\hline PPV, positive predictive value; NPV, negative predictive value. & & \\
\hline
\end{tabular}

Table 4 Screening test indices at each level OC curve (Ghana test data)

\begin{tabular}{lllll}
\hline Score & Sensitivity & Specificity & PPV & NPV \\
\hline$>0$ & $0.94(0.87,0.98)$ & $0.15(0.05,0.36)$ & $0.79(0.70,0.86)$ & $0.44(0.15,0.77)$ \\
$>1$ & $0.64(0.53,0.74)$ & $0.46(0.27,0.66)$ & $0.80(0.69,0.88)$ & $0.27(0.15,0.43)$ \\
$>2$ & $0.25(0.16,0.35)$ & $0.92(0.73,0.99)$ & $0.92(0.72,0.99)$ & $0.26(0.18,0.37)$ \\
\hline
\end{tabular}

PPV, positive predictive value; NPV, negative predictive value.

(other than yellow). Features found more frequently in bacterial than in fungal corneal ulcers were hypopyon and fibrin in the anterior chamber (table 1).

No significant differences were observed between the frequency of occurrence of an immune ring, keratic precipitates, perineural infiltrates, endothelial plaque, and flare or cells in the AC. Clinical features found to be characteristic of fungal or bacterial infection were entered into a logistic regression model.

Serrated margins, raised slough, coloration other than yellow, and fibrin were statistically independent features as determined by the logistic regression model (table 2 ).

Using the three clinical features associated with fungal infection a score was devised, with the presence of a significant feature scoring +1 . The higher the score, the greater the probability of fungal infection (fig $1 /$ table 3 ).

The probability of fungal infection if one of the clinical features was present; either a serrated margin, raised slough or coloration other than yellow; was 63\%; the presence of all three signs indicated an $83 \%$ chance of fungal infection.

The results obtained from testing the cases from Ghana closely correlated with those from the Indian dataset and the presence of all three signs resulted in a probability of $>90 \%$ that the infection was fungal (fig 2/table 4).

Approximately $17 \%$ of the corneal ulcers in the Indian dataset and $>50 \%$ of corneal ulcers in Ghana scored zero. These comprised bacterial corneal ulcers and fungal corneal

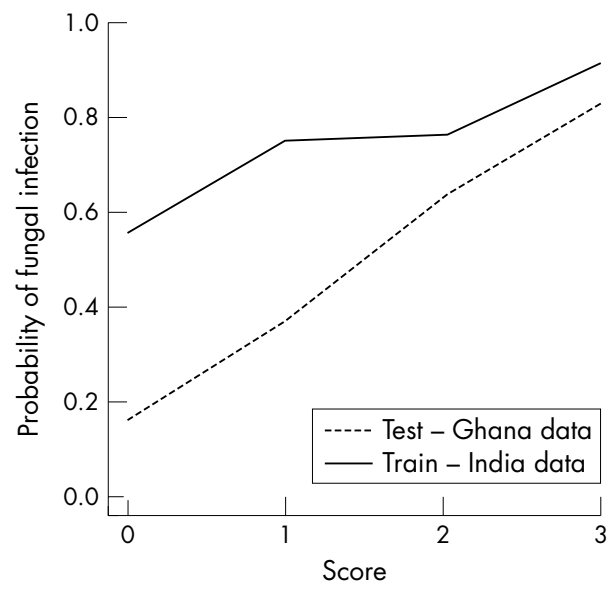

Figure 2 Operating characteristic $(O C)$ curve for diagnostic score (Ghana data). ulcers for which all of the scored clinical features were absent.

\section{DISCUSSION}

Filamentous fungi are the commonest cause of mycotic keratitis in many countries in tropical latitudes and it is vital that a specific diagnosis is made as quickly as possible to ensure prompt institution of antifungal therapy. ${ }^{6-8} 14-18$ Although a detailed clinical examination may help to reach a rapid presumptive diagnosis, fungal keratitis continues to be confused with other causes of inflammatory keratitis. ${ }^{19}$ Certain clinical characteristics of corneal ulcers may suggest a specific pathogen, but it is now generally accepted that a reliable diagnosis cannot be made by clinical appearance alone and that microbiological investigations should be performed. ${ }^{20}{ }^{21}$ Unfortunately, many ophthalmologists working in developing countries do not have access to basic ocular microbiological investigations such as microscopy or culture of corneal scrapes. Even in the United States, patients with corneal ulcers are frequently not referred for microbiological investigations and ophthalmologists tend to depend on their clinical acumen when prescribing. ${ }^{22}{ }^{23}$ Thus, it is imperative to assess the reliability of what are considered to be "characteristic" clinical features in the diagnosis of suppurative keratitis.

The observations of Kaufman and Wood and Jones are now firmly established in the ophthalmic literature, however, both of these and other similar observational studies have limitations. There was no comparison of the frequency of occurrence of these clinical features in fungal, bacterial, or other types of microbial ulcers and there was no assessment of the relative importance of these different characteristics in establishing a diagnosis. It is essential to determine the validity of these concepts, an issue which most published case series of microbial keratitis have not attempted to address. $^{6} 7141518$ 24-26

Only in recent studies have authors compared clinical features in fungal and bacterial keratitis. In a study of 142 patients with suppurative keratitis in Bangladesh, patients with culture proved fungal keratitis (almost all caused by filamentous fungi) reported a longer history of symptoms than bacterial ulcers $(\mathrm{p}<0.01)$ and a dry, raised, necrotic or fluffy surface were more frequent $(p<0.01)$. Endothelial rings were also more frequent in fungal than in bacterial ulcers, whereas dacryocystitis was significantly more common in bacterial ulcers. ${ }^{16}$ Wong et al compared fungal and bacterial keratitis in a hospital based retrospective study in Singapore and reported anterior chamber involvement to be more common in fungal ulcers $(45 \% v 35 \%) .{ }^{17}$ In many of the 
studies serrated margins are commonly reported in association with fungal infection. ${ }^{1-5}$ In our study $79 \%$ of fungal ulcers had serrated margins, but this sign was not pathognomonic, as $48 \%$ of bacterial ulcers also had serrated margins.

Another important feature of fungal corneal ulcers is believed to be raised, dry, necrotic slough ${ }^{12}{ }^{4}$ In our study dry textured slough was seen more frequently in fungal corneal ulcers. Satellite lesions, which are reported to occur frequently in fungal corneal ulcers, were also seen in cases of bacterial keratitis although less frequently occurring. ${ }^{1-3}$ An immune ring is believed to be a frequent occurrence in a filamentous fungal corneal ulcer; in the present study, an immune ring was only observed in very few cases. Anterior chamber pathology (hypopyon, fibrin) was more frequently observed in bacterial than in fungal ulcers. This is in contrast with the observations of some authors but consistent with the findings of others. ${ }^{1-3} 14172526$

An ophthalmologist confronted with a patient with suppurative keratitis may be uncertain as to which clinical features should be given more importance in differentiating between fungal and bacterial infection. Although certain clinical features appear to be more strongly associated with fungal infection we do not believe it is wise to depend on a single clinical feature to reach a presumptive diagnosis. The rationale for devising a scoring scheme was based on the premise that the simultaneous presence of several "characteristic" clinical features in a corneal ulcer should permit a more precise diagnosis of fungal infection than individual characteristics considered in isolation. If a high score is obtained then the observer can be $83 \%$ certain of fungal infection. Conversely, a low score (including zero) is associated with increased probability of bacterial aetiology.

The greatest benefit of a clinical score such as this may be to clinicians who are working in eye centres where there are no facilities for laboratory investigation. The score may be used to provide a rapid indication of the type of infection, essential in guiding treatment choice. The management of corneal ulcers using this scheme will vary depending on the setting in which it is used. For example, a clinician working in a region where fungal keratitis is thought to be common may decide to use antifungals to treat ulcers with a score of 1 or 2, whereas a clinician working in an area in which fungal keratitis is less common may reserve antifungals for ulcers with a maximum score of 3 . Choosing a low score as a guide for management as a fungal ulcer may result in unnecessary treatment of bacterial ulcers with antifungals; however, patients with very early disease would not be missed. In contrast, the selection of a highest score as a cut-off point would permit more specific diagnosis, and a more selective institution of antifungal therapy; however, this may mean that for patients with very early disease, where the clinical features may be vague and non-specific, appropriate treatment may be delayed. For ophthalmologists living in areas where fungal keratitis is a common or frequent cause of keratitis (southern India, sub-tropical Africa, and south Florida), low scoring ulcers may be given antifungals whereas for those working in more temperate climates (the United Kingdom, northern United States) the decision to treat with antifungals might be confined to those cases which score highly. The decision as to when to treat an ulcer with antifungals may also be influenced by other factors such as financial constraints and availability of antifungal agents. It is recommended that fungal infections are treated with a combination of an antibiotic and an antifungal agent in case of mixed infection.

We did not stratify clinical presentation based on the duration of the symptoms as this information was thought to be unreliable as the majority of patients reported short duration of symptoms that did not concur with the clinical picture. It is possible that if duration of symptoms were included the results might have been different. We have also used only two broad categories-namely, culture proved bacterial keratitis, and microscopy or culture proved fungal keratitis, and not introduced subsets based on the infecting fungal or bacterial genus in each category, as a larger number of cases would have been needed. However, from our results it is clear that bacterial and fungal ulcers can exhibit the same features and therefore analysing the data based on genera may reduce the usefulness of such a test.

Further investigations to test and refine this scoring scheme are currently under way and there are plans to validate the proposed scheme in different settings.

\section{CONCLUSIONS}

The clinical features of microbial keratitis may vary considerably and no one clinical feature can be considered as absolutely pathognomonic of a particular type of aetiological agent.

Ophthalmologists are urged to send corneal scrapes for microbiology examination where facilities for ocular microbiology are available. However, where such facilities are not available, a rapid presumptive clinical diagnosis of filamentous fungal keratitis may be possible using a tool such as the scoring scheme presented here. When fungal infection is suspected a combination of antibiotic and antifungal therapy is recommended.

\section{ACKNOWLEDGEMENTS}

The authors would like to acknowledge the work of the ophthalmologists and microbiologists in India and Ghana who were involved in the collection of the original data. ${ }^{8}$ This research was funded by the Community Fund, UK.

\section{Authors' affiliations}

P A Thomas, Institute of Ophthalmology, Joseph Eye Hospital, Tiruchirapalli, India

A K Leck, International Centre for Eye Health, Clinical Research Unit, London School of Hygiene and Tropical Medicine, London, UK M Myatt, Division of Epidemiology, Institute of Ophthalmology, London, UK

Competing interests: none declared

Ethical approval was obtained from the ethics committees at Moorfields Eye Hospital, Joseph Eye Hospital (India) and Korle Bu Teaching Hospital (Ghana).

\section{REFERENCES}

1 Kaufman HE, Wood RM. Mycotic keratitis. Am J Ophthalmol 1965;59:993-1000.

2 Jones BR. Principles in the management of oculomycosis. Am J Ophthalmol 1975:79:719-51.

3 Foster CS. Fungal keratitis. Infect Dis Clin N America 1992;6:851-7.

4 Thomas PA. Mycotic keratitis:an underestimated mycosis. J Med Vet Mycol 1994;32:235-54.

5 Klotz SA, Penn CC, Negvesky GJ, et al. Fungal and parasitic infections of the eye. Clin Microbiol Rev 2000;13:662-85.

6 Rosa RH Jr, Miller D, Alfonso E. The changing spectrum of fungal keratitis in South Florida. Ophthalmology 1994;101:1005-13.

7 Garg P, Gopinathan U, Choudhary K, et al. Keratomycosis-clinical and microbiologic experience with dematiaceous fungi. Ophthalmology 2000;107:574-80

8 Leck AK, Thomas PA, Hagan M, et al. Aetiology of suppurative corneal ulcers in Ghana and south India, and epidemiology of fungal keratitis. Br J Ophthalmol 2002;86:1211-15.

9 Jones DB. Strategy for the initial management of suspected microbial keratitis. In: Barraquer J, Binder PS, Buxton JN, et al, eds. Transactions of the New Orleans Academy of Ophthalmology. Symposium on Medical and Surgical Diseases of the Cornea. St Louis: CV Mosby, 1980:86-119.

10 Gilman J, Myatt M. Epicalc 2000 Ver 1.02, Brixton Books, 1998.

11 Cleveland WS. Robust locally weighted regression and smoothing scatterplots. J Am Statist Assoc 1979;74:829-36.

12 Cleveland WS. LOWESS: a program for smoothing scatterplots by robust locally weighted regression. Am Stat 1981;35:54. 
13 Ihaka R, Gentleman R. R: a language for data analysis and graphics. J Comput Graph Statist 1996;5:299-314.

14 Gugnani HC, Talwar RS, Njoku-Obi ANU, et al. Mycotic keratitis in Nigeria. A study of 21 cases. Br J Ophthalmol 1976;60:607-13.

15 Liesegang TJ, Forster RK. Spectrum of microbial keratitis in South Florida. Am J Ophthalmol 1980;90:38-47.

16 Dunlop AA, Wright ED, Howlader SA, et al. Suppurative corneal ulceration in Bangladesh: a study of 142 cases, examining the microbiological diagnosis, clinical and epidemiological features of bacterial and fungal keratitis. Aust NZ J Ophthalmol 1994;22:105-10.

17 Wong T-Y, $\mathrm{Ng} \mathrm{T-P}$, Fong K-S, et al. Risk factors and clinical outcomes between fungal and bacterial keratitis: a comparative study. CLAO J 1997:23:275-81.

18 Gopinathan U, Garg P, Fernandes $M$, et al. The epidemiological features and laboratory results of fungal keratitis. A 10 year review at a referral eye care center in South India. Cornea 2002;21:555-9.
19 Sridhar MS, Gopinathan U, Garg P, et al. Aspergillus fumigatus keratitis with wreath pattern infiltrates. Cornea 2001;20:534-5.

20 Florakis GJ, Moasami G, Schubert H, et al. Scanning slit confocal microscopy of fungal keratitis. Arch Ophthalmol 1997;115:1461-3.

21 Winchester K, Mathers WD, Sutphin JE. Diagnosis of Aspergillus keratitis in vivo with confocal microscopy. Cornea 1997;16:27-31.

22 McDonnell PJ, Nobe J, Gauderman WJ, et al. Community care of corneal ulcers. Am J Ophthalmol 1992;1 14:531-8.

23 McLeod SD, DeBacker CM, Viana MA. Differential care of corneal ulcers in the community based on apparent severity. Ophthalmology 1996; 103:479-84

24 Wilhelmus KR, Jones DB. Curvularia keratitis. Trans Am Ophth Soc 2001;99:111-32.

25 Cruz OA, Sabir SM, Capo H, et al. Microbial keratitis in childhood. Ophthalmology 1993;100:192-6.

26 Ormerod LD, Hertzmark E, Gomez DS, et al. Epidemiology of microbial keratitis in southern California. Ophthalmology 1987;94:1322-33.

\section{bmjupdates+}

bmiupdates+ is a unique and free alerting service, designed to keep you up to date with the medical literature that is truly important to your practice.

bmjupdates+ will alert you to important new research and will provide you with the best new evidence concerning important advances in health care, tailored to your medical interests and time demands.

Where does the information come from?

bmjupdates+ applies an expert critical appraisal filter to over 100 top medical journals A panel of over 2000 physicians find the few 'must read' studies for each area of clinical interest

Sign up to receive your tailored email alerts, searching access and more...

www.bmjupdates.com 